\title{
DYNAMICS OF LIQUID METAL JETS PENETRATING A STRONG MAGNETIC FIELD IN HIGH-POWER COLLIDERS
}

\author{
A. Hassanein and I. Konkashbaev \\ Argonne National Laboratory, Argonne, IL, USA
}

\begin{abstract}
One very important problem of future high-power colliders, such as the muon collider, is to create an effective and reliable target system that is able to withstand a harsh environment and generate effective high-flux muon beams. Liquid metal jets (pulsed or continuous) are proposed as potential target candidates. Such a proposal poses two critical problems: penetration of a free liquid jet inside the required strong inhomogeneous magnetic field $(20 \mathrm{~T})$ and the fragmentation of liquid due to the intense sudden heating by the proton beam and the resultant magnetohydrodynamic instabilities. In this study, we analyzed the dynamic motion of a free liquid metal jet entering a strong inhomogeneous field by using a new two-dimensional numerical model implemented in the HEIGHTS package for arbitrary jet parameters.
\end{abstract}

\section{INTRODUCTION}

To realize a $\mu^{+} \mu^{-}$collider, high-Z targets are necessary [1]. Solid targets made from tungsten, platinum, or lead would be a good choice, but the heat load could not be easily removed and the stresses from thermal-shock wave propagation can seriously damage the target. Therefore, more sophisticated targets, such as a tungsten powder flow or rotating band, are suggested [1]. Rapidly flowing liquid metal targets have been considered an option because the heat energy can be removed along with the moving liquid [1]. Theoretical and experimental studies of liquid metal target flow in magnetic field are underway.

There are mainly three important problems of using liquid metal targets in these environments. First, as the liquid jet penetrates the magnetic field of the cylindrical coil, perturbations in jet motion and deceleration can because of the large field gradients at the entrance and exit of the solenoid and because of $\mathrm{J} \times \mathrm{B}$ forces [2]. Second, during the intense pulse of energy deposited in a short time (a few ns), the strong resultant stress waves could damage the target and adjacent components [3]. Third, the liquid jet can develop instabilities during both liquid motion in the strong inhomogeneous magnetic field and after beam energy deposition because of RayleighTaylor and magnetohydrodynamic (MHD), balloon mode, instabilities. These instabilities can change the jet shape into a significantly less efficient target for pion production. Tolerance to capillary instabilities can be achieved by an improved nozzle shape that has been proved experimentally for lithium and water jets [4].

\section{BEAM/TARGET SYSTEM}

The parameters of the proton beam and solenoid coil system are determined by the required conditions of pion and muon production rates [1]. Basic system parameters consist of a proton energy $\mathrm{E}_{\mathrm{p}}=16 \mathrm{GeV}$, number of protons in one bunch $\mathrm{N}_{\mathrm{p}} \approx 10^{15}$, bunch beam power of 280 $\mathrm{kW}$, with frequency $\mathrm{f}=15 \mathrm{~Hz}$ and total power of $4 \mathrm{MW}$. Only $10 \%$ of the beam power is absorbed inside the target. The solenoid has a length $\mathrm{L}_{\text {coil }}=20 \mathrm{~cm}$, radius $\mathrm{R}_{\text {coil }}=10 \mathrm{~cm}$, and a maximum magnetic field $\mathrm{B}_{\mathrm{o}}=20 \mathrm{~T}$. The beam arrives at an angle $\alpha_{\text {beam }}=100$ millirad and interacts with the liquid jet of radius $\mathrm{R}_{\mathrm{O}} \approx 0.5 \mathrm{~cm}$, coming at an angle $\alpha_{\text {jet }}=67$ millirad; therefore, the angle between the beam and the jet is $\alpha=\alpha_{\text {beam }}-\alpha_{\text {jet }}=33$ millirad $=$ $1.89^{\circ}$, as schematically shown in Fig. 1.

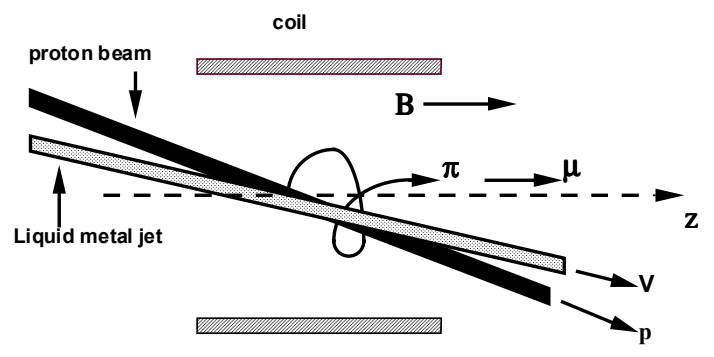

Fig. 1. Beam/liquid jet interaction system

The required jet velocity $\mathrm{V}$ is determined by two conditions: first, $\mathrm{V}$ should be high enough to remove the energy deposited by the proton beam, and second, it should be high enough to overcome the deceleration force $\vec{F}=[\vec{j} \times \vec{B}]$. For a mercury jet with $\mathrm{R}_{\mathrm{o}} \approx 0.5 \mathrm{~cm}$, the radial Reynolds magnetic number $\operatorname{Re}=4 \pi \sigma V R^{2} /\left(c^{2} L\right)<<1$; thus, the magnetic field of the induced current can be neglected but the force $F$ from the induced electric field $E$ and corresponding current $j$, where

$$
\vec{E}=[\vec{V} \times \vec{B}], \vec{j}=\sigma \vec{E}=\sigma[\vec{V} \times \vec{B}],
$$

should be taken into account.

The jet motion along the r-direction leads to jet compression that tends to instantly establish an equilibrium pressure at any $\mathrm{z}$ with a distribution $\operatorname{Pr}(\mathrm{z}, \mathrm{r})$. 
This pressure leads to the first deceleration force " $\theta$-pinch effect" [3];

$$
F_{r z}=-\nabla_{z} P_{r}(r, z), P_{r}(r, z)=\int_{0}^{r} \rho F_{r} d r
$$

$\mathrm{P}_{\mathrm{r}}(\mathrm{r}, \mathrm{z})$ has a maximum absolute value $\mathrm{P}_{\max }$ at a distance near both the coil entrance and exit, which determine the minimum velocity necessary at the axis to overcome this pressure. For a stationary flow, it is possible to define flow "potential" $\Psi$ as

$$
\Psi=P_{r}+P_{z}, \quad P_{z}(r, z)-\int_{o}^{z} F_{z}(r, \xi) d \xi
$$

The solution of the Bernoulli equation

$$
\rho V^{2}+2 \Psi(z)=\rho V_{0}^{2}
$$

predicts the evolution of the jet velocity streamlines coming at various distances $\mathrm{R}$, from the axis, as shown in Fig. 2. Practically, the velocity loss at boundary streamlines does not depend on initial jet velocity $\mathrm{V}_{\mathrm{o}}$. For example, $\Delta \mathrm{V}\left(\mathrm{R}_{\mathrm{o}}=1 \mathrm{~cm}\right)=21.7 \mathrm{~m} / \mathrm{s}$; therefore, a jet with a velocity of $\approx 20 \mathrm{~m} / \mathrm{s}$ will not pass through a solenoid with $\mathrm{B}_{\mathrm{o}}=20 \mathrm{~T}$.

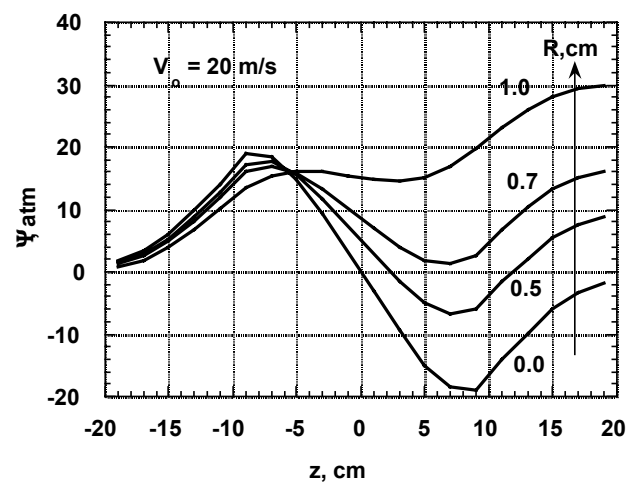

Fig. 2. Streamline potential $\Psi(\mathrm{R}, \mathrm{z})$

\section{NUMERICAL SIMULATION}

The MHD equations of jet motion are fully solved by using a two-dimensional hybrid Eulerian-Lagrangian numerical scheme implemented in the HEIGHTS package [5-6]. Calculations were made for various values of jet velocity, radius, and magnetic field. Figures 3 and 4 show the axial velocity and streamlines for a mercury jet with initial velocity $\mathrm{V}_{0}=20 \mathrm{~m} / \mathrm{s}, \mathrm{B}_{0}=20 \mathrm{~T}$, and $\mathrm{R}_{0}=0.5 \mathrm{~cm}$ (basic design). Figure 5 shows the dependence of velocity loss $\Delta \mathrm{V}$ on minimum velocity $\mathrm{V}_{\min }$, ratio of maximum outgoing radius to incoming radius $R / R_{0}$, and the parameter $\Delta \mathrm{V} / \mathrm{R}$.

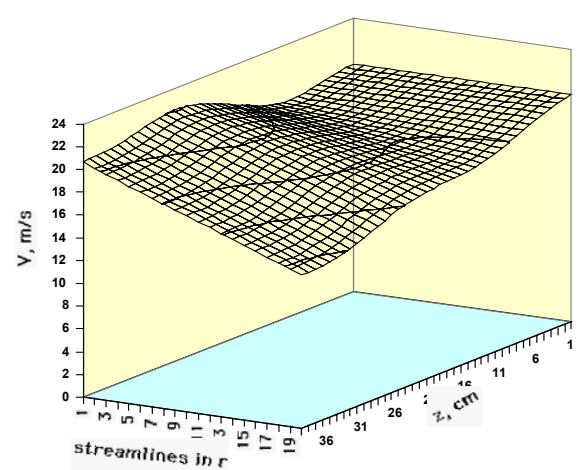

Fig. 3. Axial velocity $\mathrm{V}_{\mathrm{z}}(\mathrm{r}, \mathrm{z})$ of streamlines in Lagrangian coordinate

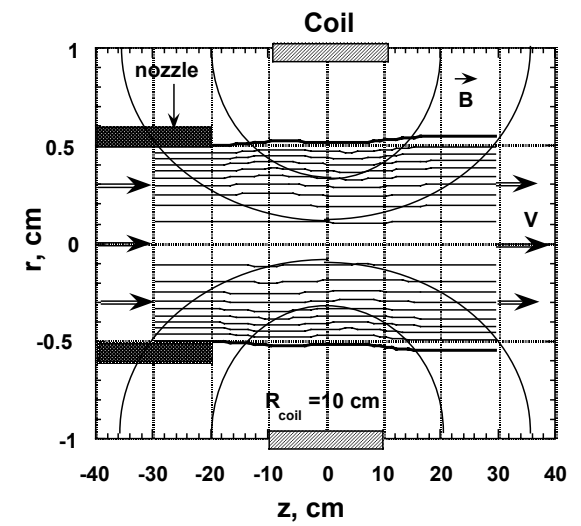

Fig. 4. Jet flow and shape inside solenoid

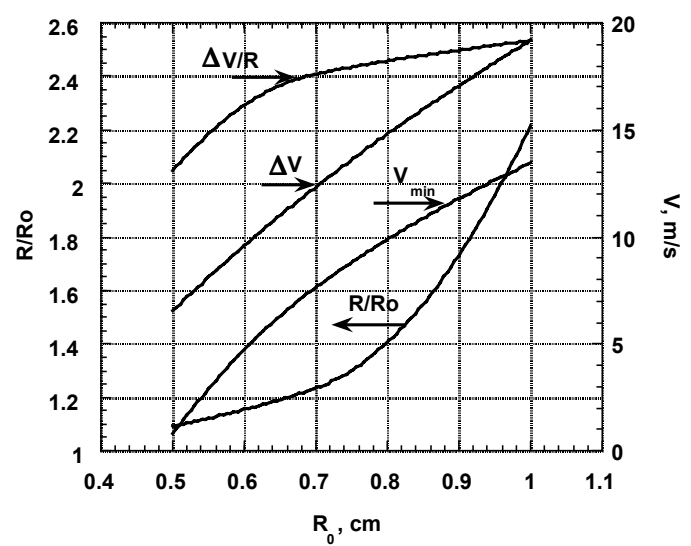

Fig. 5. Dependence of velocity loss on minimum velocity, ratio $\Delta \mathrm{R} / \mathrm{R}$, and $\Delta \mathrm{V} / \mathrm{R}$

\section{CONCLUSIONS}

The main problems of liquid metal jets penetrating a strong field are velocity loss and jet radius oscillation. As indicated from analytical estimates that are confirmed by detailed numerical calculations, the magnitudes of the braking forces $\mathrm{F}_{\mathrm{r}}$ and $\mathrm{F}_{\mathrm{Z}}$ are determined by the magnetic field near the jet surface.

Decreasing the magnetic field strength leads to decreasing oscillations of the jet radius and velocity loss. 
Increasing jet velocity leads to increased induced electric field, corresponding current, and stopping forces. Although the velocity loss $\Delta \mathrm{V}$ increases with $\mathrm{V}_{\mathrm{o}}$ (e.g., from $20 \mathrm{~m} / \mathrm{s}$ to $25 \mathrm{~m} / \mathrm{s}$ ), the relative velocity $\operatorname{loss} \xi=\Delta V / V_{O}$ decreases from $\approx 1$ to 0.5 and leads to decreasing relative changes in jet radius $\zeta=\Delta R / R_{O} \propto \xi$. Oscillations of jet radius decrease with initial jet velocity.

A decrease in jet radius means a decrease in the radial magnetic field acting on the jet; thus, the absolute $(\Delta \mathrm{V}=$ $6.5 \mathrm{~m} / \mathrm{s})$ and relative $(\xi \leq 3 \%)$ velocity loss decrease as do relative changes in jet radius $(\zeta<3 \%)$. These values seems reasonable for the current optimized parameters with $\mathrm{R}_{\mathrm{o}}=0.5 \mathrm{~cm}, \mathrm{~V}_{\mathrm{o}}=20 \mathrm{~m} / \mathrm{s}$, and beam radius $\mathrm{R}_{\mathrm{p}}=$ $0.15 \mathrm{~cm}$.

\section{ACKNOWLEDGMENTS}

Work is partially supported by the U.S. Department of Energy, Office of High-Energy Physics.

\section{REFERENCES}

[1] J. Alessi et al., A proposal for an R\&D program for targetry and capture at a muon-collider source. (Sept.
28, 1998), approved as BNL E951, http: /www. hep. Princeton. edu/ $\sim$ mcdonald/mumu/target/targetprop.pdf

[2] P. Thieberger, Brookhaven National Laboratory, Nov. 4, 2000, Harold G. Kirk, Targetry and capture for the muon collider, BNL, in Muon Collider Collaboration Meeting, Berkeley, CA, Oct. 8, 1998. Also: $\mathrm{ftp}: / / \mathrm{ftp}$.numi-study2.bnl.gov/pt/

[3] A. Hassanein, I. Konkashbaev, J. Norem, "Thermoelastic response of suddenly heated liquid and solid targets for high-power colliders," These proceedings.

[4] A. Konkashbaev, N. B. Morley, K. Gulec, T. Sketchley, Stability and contraction of a rectangular liquid metal jet in a vacuum environment. Fusion Eng. Des., 51-52 (2000), pp.1109-1114.

[5] A. Hassanein, "Liquid Metal Targets for High-Power Applications: Pulsed Heating and Shock Hydrodynamics," Laser \& Particle Beams, Vol. 18, 2000 , p. 611.

[6] A. Hassanein and I. Konkashbaev, Numerical simulation of liquid metal jet penetrating a strong magnetic field, Argonne National Laboratory Report ANL-ET/01, June 2001. 